\title{
Evaluation of argon plasma coagulation in healing of a solitary rectal ulcer in comparison with conventional therapy: a randomised controlled trial
}

\author{
Farzad Jassemi Zergani, Ali Akbar Shaiesthe, Eskandar Hajiani, Jalal Hashemi, Rahim Masjedizadeh, \\ Vaheid Sebghatollahei, Pejman Alavinejad, Ahmad Kadkhodaei, Kambeiz Akhavan, Saeid Seyyedian \\ Hepatology Department, School of Medicine, Jundishapur University of Medical Sciences, Ahvaz, Iran
}

Gastroenterology Rev 2017; 12 (2): 128-134

DOI: https://doi.org/10.5114/pg.2016.64846

Key words: solitary rectal ulcer syndrome, argon plasma coagulation, conventional therapy, behaviour therapy.

Address for correspondence: Farzad Jassemi Zergani, Hepatology Department, School of Medicine, Research Institute for Infectious Diseases of the Digestive System, Jundishapur University of Medical Sciences, Ahvaz, Iran, e-mail: fjassemi49@yahoo.com

\begin{abstract}
Introduction: Argon plasma coagulation (APC) has been reported to be effective in the treatment of solitary rectal ulcer syndrome (SRUS). However, it has not appeared to be effective in healing ulcers.

Aim: This study aimed at assessing the effectiveness of APC in controlling rectal ulcer-induced bleeding, and at examining the ultimate effect of this approach in healing these lesions.

Material and methods: This randomised, controlled trial was conducted on 99 patients with SRUS. Patients were randomly enrolled into two groups of APC therapy (intervention) and conventional therapy (control). The control group $(n=58)$ received a high-fibre diet, laxatives, behaviour therapy, and sucralfate enemas, and the intervention group $(n=41)$ were treated with APC plus conventional therapy; in fact they received directed and focused argon gas in addition to a high-fibre diet and laxatives.

Results: Responses to treatment in the control group and in the APC-receiving group were $29.3 \%$ and $75.6 \%$, respectively. The continuation of ulcer healing after 3 months in the control group was $10.3 \%$, and it was $70.7 \%$ in the APC-treated group. There was a significant statistical difference between the two groups $(p<0.004)$, i.e. bleeding was controlled more frequently in the group receiving APC plus conventional therapies than in the group receiving only the conventional therapies. However, the results showed no statistically significant difference between the two groups in terms of pain relief $(p<0.36)$.

Conclusions: Argon plasma coagulation not only controlled bleeding in patients with SRUS, but also, in comparison with the conventional methods of treating SRUS, led to healing and continuation of healing of rectal ulcers.
\end{abstract}

\section{Introduction}

Solitary rectal ulcer syndrome (SRUS) is an uncommon, benign, and often undetectable disease, which usually involves the rectum [1]. Solitary rectal ulcer syndrome can affect all ages and both sexes [2]. It usually occurs in the third decade of life in men and in the fourth decade of life in women [3]. The symptoms include bloody discharge, mucus passage, and a sense of incomplete evacuation [4]. Abnormal and excessive straining during bowel movements damages the rectum [5]. The prevalence of SRUS is approximately 1 per 100,000 people per year [3]. Its nature and pathogenesis has not been fully described; however, multiple factors may be involved. One of the accepted theories is direct trauma, local ischaemia, and rectal mucus [6]. The most common site of ulcers is the anterior wall of the rectum, 7 to $10 \mathrm{~cm}$ above the anal verge [7].

Diagnosis is made usually based on clinical, endoscopic, and histological findings [5]. Symptoms may subside spontaneously, but most patients ultimately need medical or surgical treatment. The main and primary treatments include training patients (evacuation behaviour), family support, preventive measures such as high-fibre diet, and behavioural therapy (toilet manners). Some special treatments include sucralfate, salicylate, corticosteroid, and injection of corticosteroids or botulinum toxin into or around the ulcers, which is effective in the treatment of minor ulcers. Results have shown that the majority of patients do not respond definitively to the conventional therapies (behavioural therapy, biofeedback, topical treatments, and surgery) [8]. In a study 
conducted on 23 SRUS patients, 7 received medical treatment, among which, only 3 were fully healed after the treatment. Other patients were treated surgically, among whom, symptoms returned after surgery in 4 cases, and 2 others needed further surgical interventions. Also, 3 patients were diagnosed with sexual dysfunction, and one still suffered from pain in the anus after colostomy [9]. A study conducted on 6 patients treated with sucralfate enema ( $2 \mathrm{~g} /$ day for 6 weeks) reported macroscopic healing in all SRUS patients after 8 months of follow-up [10]. The results of these studies suggest that these treatments do not result in complete healing. However, in the recent years the use of new treatment methods in various diseases has attracted academic interest.

Argon plasma coagulation (APC) is a newly proposed therapeutic approach for the treatment of SRUS. A non-contact electrocoagulation device is used in this treatment. This device utilises a high-frequency mono-polar current directed by ionised argon gas to coagulate tissues and mucosal ulcers. Argon plasma coagulation may heal ulcers through squamous regeneration and re-epithelialisation mechanism [10]. Few studies are available about the effectiveness of APC compared to conventional therapy. For example, Somani et al. studied 24 patients in 2010 in India. Twelve patients were treated with biofeedback (BT) and 12 with APC. Out of the 12 APC-treated patients, 8 were fully healed after four to eight treatment sessions, while only 2 of the 12 BT-treated patients were improved [5].

Since APC-therapy has been investigated in only two or three studies worldwide, the method seems to be very new. Given the low number of studies on the effectiveness of APC compared to conventional therapy, and due the acceptable effectiveness of APC reported in a number of articles, the authors decided to conduct a study to compare the effectiveness of APC with conventional therapy in the healing of rectal bleeding ulcers in SRUS patients.

\section{Aim}

The objective of this study was to examine the effect of APC in controlling and healing the ulcers in SRUS patients and to investigate the harmlessness of this approach. APC was also compared with the conventional treatment methods.

\section{Material and methods}

\section{Study design and population}

This randomised, controlled clinical trial was performed in 2010 in Imam Khomeini Hospital of Ahwaz on 99 patients with previously diagnosed SRUS based on endoscopic symptoms and pathologic and histologic findings. Patients underwent CBC-diff test, liver tests (aspartate aminotransferase (AST), alanine aminotransferase (AST), alkaline phosphatase (ALP)), coagulation profile (prothrombin time (PT), partial thromboplastin time (PTT), international normalised ratio (INR)), erythrocyte sedimentation rate (ESR), C-reactive protein (CRP), as well as colonoscopy and proctosigmoidoscopy, to rule out other causes of rectal bleeding and comorbidities.

\section{Inclusion criteria}

Inclusion criteria were SRUS ulcer and histological diagnosis by a pathologist.

\section{Exclusion criteria}

Malignant ulcers and wounds that histologically lacked the SRUS nature were excluded.

\section{Ethical considerations}

A questionnaire was designed and the patients completed it before entering the study. Written consent was obtained from all subjects. The nature and process of treatment was fully described to all patients or their families.

\section{Procedure}

The research was an experimental randomised, controlled trial. The studied population consisted of all patients with SRUS referring to the Imam Khomeini Hospital of Ahwaz during 2010-2013. Inclusion criteria were ulcers with SRUS characteristics, which were histologically diagnosed by a pathologist. Exclusion criteria were malignant ulcers and wounds that histologically lacked the SRUS nature.

The sample size was 99 (58 patients were placed in the conventional therapy group, and 41 in the APC-plus-conventional-therapy group). The original sample size was 115, among which, 15 patients from the intervention group, and one patient from the control group refused to return for follow-ups. Thus, these 16 patients were excluded. Laboratory tests (CBC-diff), liver tests (AST, ALT, ALP), coagulation profile (PT, PTT, INR), ESR, CRP, and S/E were requested for the patients. All subjects also underwent total colonoscopy or proctosigmoidoscopy (only if they had undergone a total colonoscopy) to diagnose SRUS and to rule out other causes of rectal bleeding and comorbidities.

The questionnaire was completed by the patients at admission and during follow-up sessions in the digestive clinic of Imam Khomeini Hospital of Ahwaz. Before completing the questionnaire, their consent to enter the trial was obtained from all patients. Further details about the nature of treatment and its stages were giv- 
en to all patients or their families. After determining the nature, size, and number of the SRUS ulcers, the patients were randomly (through even or odd numbers) divided into the intervention group (APC plus conventional therapy) or the control group (conventional therapy). The number of ulcers, their distance from the anal verge, their size, and whether or not they were polypoid were recorded in a checklist during proctosigmoidoscopy. The ulcers characteristics were also recorded in a table after each session of APC or after an outpatient visit with proctosigmoidoscopy.

Conventional treatments in the control group consisted of behavioural therapy, sucralfate enemas, highfibre diet, and psyllium powder. All patients in the control group were visited in 4-week intervals for an average of six sessions as outpatients. Each visit lasted 15 to $30 \mathrm{~min}$. The behavioural therapy (BT) used in this study encompassed a discussion about the nature of the defecation, consumption of a high-fibre diet, and toilet manner. All patients were recommended to use European toilets rather than squat toilets. All patients were advised to use the muscles of the abdominal wall for evacuation. Patients were asked to refrain from frequent use of toilet (in order to reduce the straining time during evacuation) and not to use their fingers for evacuation (digital evacuation). They were also asked to ignore the misconceptions about the "sense of incomplete evacuation in most patients". It was explained to them that this feeling is caused by chronic prolapse of the rectal mucus and pressure on the perineum. Coloured anatomical figures were used to better illustrate the trainings.

All patients were also treated with sucralfate enemas (4 tablets of $500 \mathrm{mg}$ powdered in $15 \mathrm{ml}$ saline) every night before bed, and psyllium powder one to three times a day in the case of constipation.

All patients receiving conventional treatment underwent proctosigmoidoscopy to observe rectal ulcers after six visits with 4-week intervals. In the case of ulcer healing, this time was considered the end point of treatment. If the ulcers were not healed and the signs of bleeding were observed at endoscopy after six visits, the patient was considered as a non-responder. The patient healed with conventional treatments, and who finished the treatment, underwent proctosigmoidoscopy 3 months later in order to prove the continuation of healing or recurrence of ulcers.

In the group treated with APC plus conventional therapy, APC was applied as outpatient in 4-week intervals aimed at controlling bleeding and healing the ulcers. In this group, behavioural therapy, high-fibre diet, and drinking plenty of fluids, use of laxatives, and sucralfate enemas were the main treatments. A maximum of six sessions of APC were conducted.
If the ulcers were not healed after six sessions, the ulcer was considered as a failure to treat. If the ulcers were healed after six APC sessions, this time point was considered the end of treatment for this group of patients. To examine the continuation of healing or recurrence of the ulcers, the group underwent proctosigmoidoscopy 3 months after responding to therapy. Patients treated with APC were simultaneously compared to those receiving conventional therapy. Argon plasma coagulation was conducted using a $7 \mathrm{Fr}$ probe connected to an argon generating device (EREB). To coagulate ulcers, argon was sprayed onto them without direct contact. The device was set as follows: power $40 \mathrm{~W}$; Argon gas flow $2 \mathrm{l} / \mathrm{min}$; the distance between the APC probe and the endoscope tip about $1 \mathrm{~cm}$; and distance between the probe and the ulcer and mucus was maximally 1 to $2 \mathrm{~mm}$ without direct contact. The basic principles of treatment in this group included behavioural therapy, consumption of plenty of fluids, high-fibre diet, use of bulk-forming laxative, and 5-ASA enema (Figure 1).

In all endoscopy sessions, the characteristics of ulcer, ulcer size, number of ulcers, and stigmata of bleeding were recorded in a table during the session, and the course of treatment was evaluated accordingly.

\section{Data analysis}

All the obtained data were then analysed using SPSS-19. Independent $t$-test was used to compare the quantitative variables (e.g. healing time) and $\chi^{2}$ test to compare the rates of healing. The level of statistical significance in all tests was 0.05 .

\section{Results}

The mean age of the APC-treated patients was $30.73 \pm 14.75$ years. The mean age of the patients treated with conventional therapy was $31.78 \pm 13.96$ years. The age range in both groups was $31-32$ years. In both groups, $56 \%$ were male and $43 \%$ were female. Twenty-one percent of the patients used European toilets, and $78 \%$ used squat toilets. Approximately, 30\% of patients had mental disorders and were being treated with medication. The frequency of bowel movements varied between once a day and once every 4 days. The straining time varied between 3 to $6 \mathrm{~min}$. The mean straining time was $5.56 \mathrm{~min}$. More than $55 \%$ of patients had mucus passage, and $60 \%$ used digital evacuation.

Approximately $76 \%$ of the patients had a sense of incomplete evacuation, and nearly half of them had pain around the anus.

Sixty-seven percent of the SRUS patients were not taking certain medications (e.g. NSAIDs, cardiovascular 


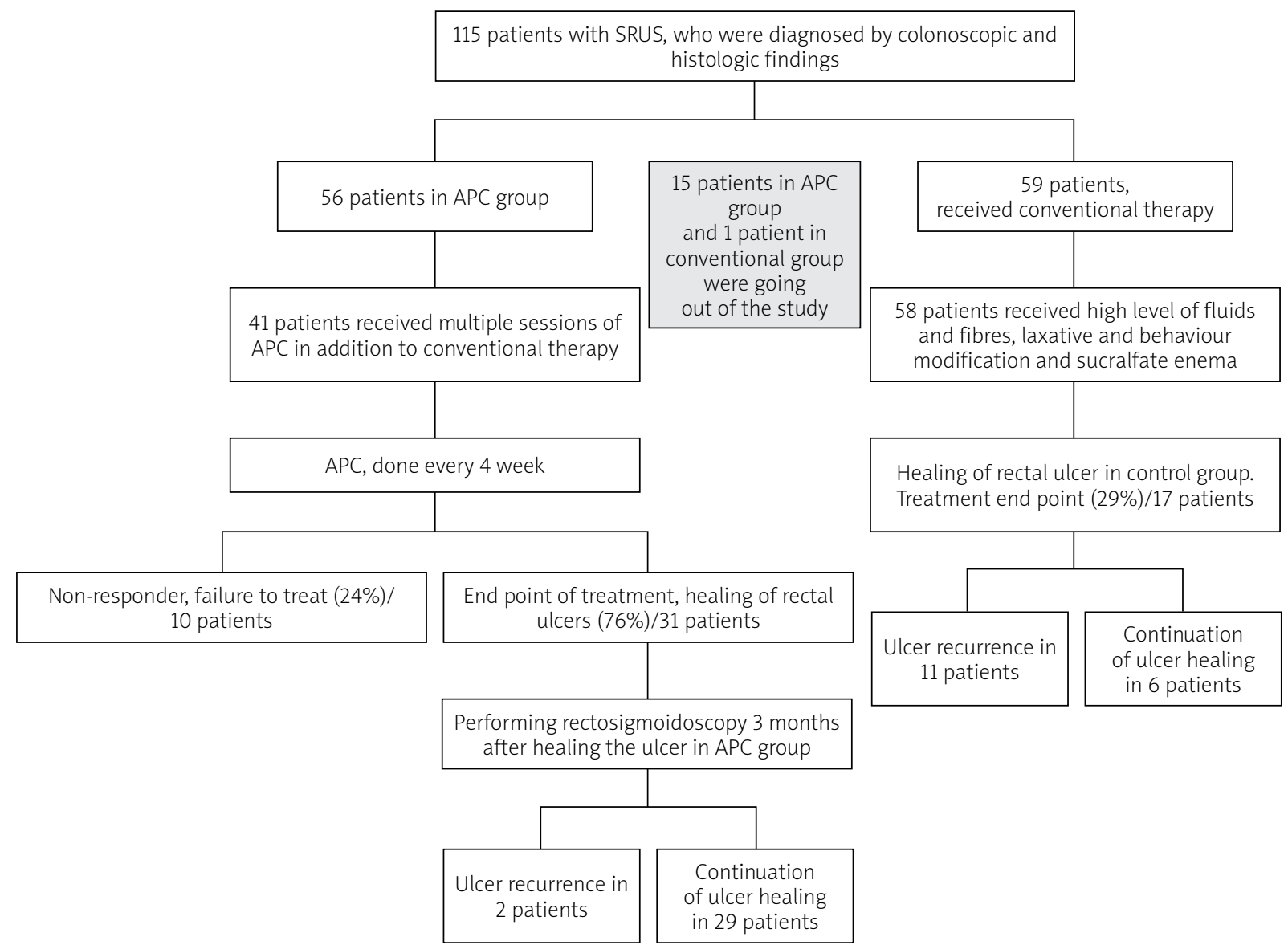

Figure 1. Algorithm of methodology

medications, aspirin, Plavix, and antidepressants), and $93 \%$ of patients did not have comorbidities.

The diameter of stool was reduced only in $15 \%$ of cases. The distance from rectal ulcer and the anal verge was within 5 to $8 \mathrm{~cm}$ in $60 \%$ of cases. The ulcer shape was flat in $79 \%$ of the cases and polypoid in $21 \%$ of them. The mean number of APC therapy sessions was five to six sessions. The timeframe between the onset of symptoms and the diagnosis of SRUS was 1 to 9 years. In fact, the mean timeframe between the onset of symptoms and the diagnosis of SRUS was about 4 years $(4.01 \pm 1.89)$. Ulcers formed more than once in $45 \%$ of the SRUS patients.

Regarding the characteristics of the ulcer, $72 \%$ had clean base, $16 \%$ had bleeding, and $11 \%$ had only erythema in proctosigmoidoscopy (Table I and Figure 2).

Response to treatment in the control group and in the APC-receiving group was $29.3 \%$ and $75.6 \%$, respectively. The continuation of ulcer healing after 3 months in the control group was $10.3 \%$, and in the APC-treated group it was approximately $70.7 \%$ (Figure 3 ).

Test results showed a significant difference between the responses to treatment. Thus, it can be said with
95\% certainty that a significant difference existed between the group receiving conventional therapy and the group receiving APC plus conventional therapy, so the effectiveness of APC was more than conventional therapy in SRUS $(p<0.001)$.

Test results showed also a statistically significant difference between the two groups regarding the continuation of ulcer healing (Table II, Figure 4). The continuation of ulcer healing was more in the APC-receiving group compared to the group receiving conventional therapy $(p<0.001)$. The results showed no significant differences between the two groups regarding pain relief ( $p<0.36)$ (Table III). Proctosigmoidoscopy in post-treatment follow-up showed no APC-induced complications in the 41 patients treated with it (Table III, Figure 5).

\section{Discussion}

The results showed that the response to treatment in the conventional therapy group was $29 \%$. It was $76 \%$ in the group receiving APC plus conventional therapy. The continuation of ulcer healing after 3 months in the control group and APC-treated group was $10 \%$ 
Table I. Frequency of ulcer characteristics

\begin{tabular}{|c|c|c|}
\hline Variables & Frequency & Percentage \\
\hline \multicolumn{3}{|l|}{ No. of wound: } \\
\hline 1 & 3 & 54.6 \\
\hline 2 & 24 & 24.7 \\
\hline 3 & 10 & 10.3 \\
\hline 4 & 4 & 4.1 \\
\hline 5 & 4 & 4.1 \\
\hline 7 & 2 & 2.1 \\
\hline Total & 99 & 100 \\
\hline \multicolumn{3}{|c|}{ Wound characteristics: } \\
\hline Bleeding & 16 & 16.2 \\
\hline Clean based & 72 & 72.7 \\
\hline Erythema & 11 & 11.1 \\
\hline Total & 99 & 100 \\
\hline \multicolumn{3}{|l|}{ Ulcer diameter: } \\
\hline 1 & 31 & 32 \\
\hline 2 & 33 & 34 \\
\hline 3 & 19 & 19.6 \\
\hline 4 & 6 & 6.2 \\
\hline Circumferential & 10 & 9.1 \\
\hline Total & 99 & 100 \\
\hline \multicolumn{3}{|l|}{ Shape of wound: } \\
\hline Flat & 79 & 79.8 \\
\hline Polypoid & 20.2 & 20.2 \\
\hline Total & 99 & 100 \\
\hline
\end{tabular}

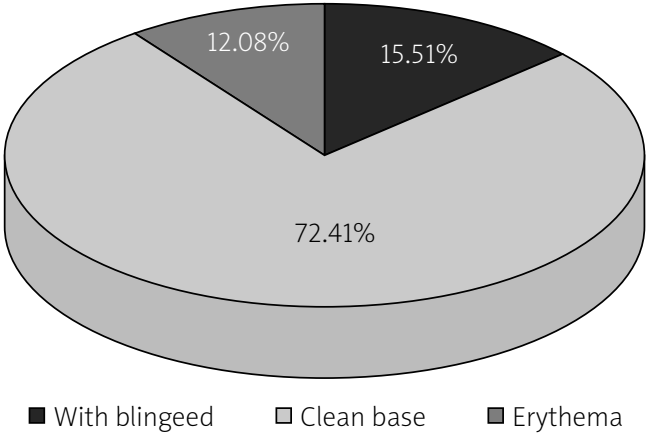

Figure 2. Characteristics of ulcer

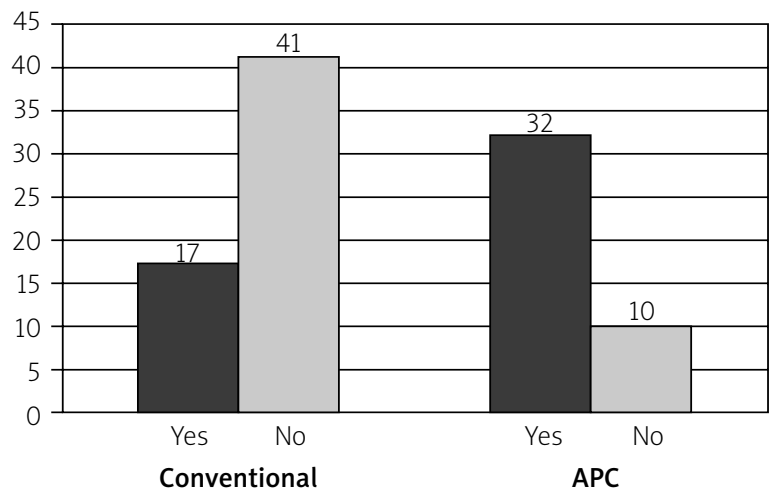

Figure 3. Response to treatment in the two groups

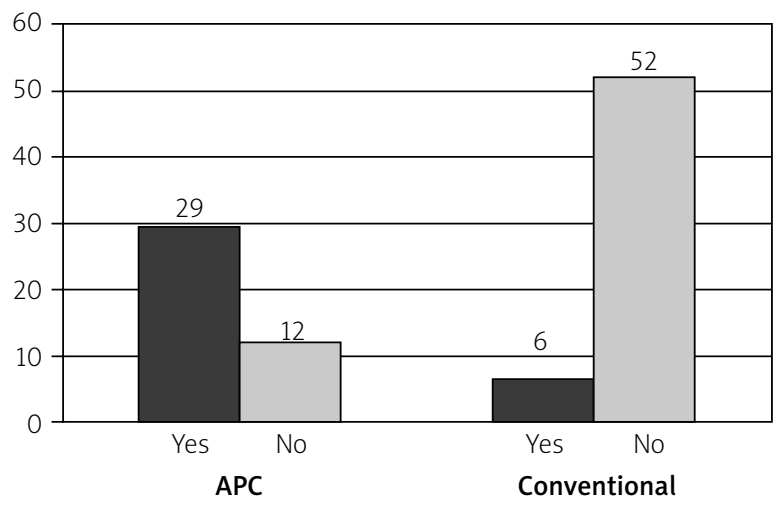

Figure 4. Comparison of continuation of ulcer healing in both groups

Table II. Comparison of the continuation of ulcer healing in the two groups of SRUS patients referred to Imam Khomeini Hospital of Ahwaz during the period 2010-2014, treated with APC and conventional therapies

\begin{tabular}{|c|c|c|c|c|c|c|c|}
\hline \multirow[t]{3}{*}{ Therapy } & \multicolumn{6}{|c|}{ Continuation of healing } & \multirow[t]{3}{*}{$P$-value } \\
\hline & \multicolumn{2}{|c|}{ Yes } & \multicolumn{2}{|c|}{ No } & \multicolumn{2}{|c|}{ Sum } & \\
\hline & $n$ & $\%$ & $n$ & $\%$ & $n$ & $\%$ & \\
\hline Treated with conventional therapies & 6 & 10.3 & 52 & 89.7 & 58 & 100 & $<0.001^{*}$ \\
\hline Treated with APC & 29 & 70.7 & 12 & 29.3 & 41 & 100 & \\
\hline Total & 35 & 35.4 & 64 & 64.6 & 99 & 100 & \\
\hline
\end{tabular}

*Significant, APC - argon plasma coagulation. 
Table III. Comparison of clinical characteristics of the two groups of SRUS patients referred to Imam Khomeini Hospital of Ahwaz during the period 2010-2014, treated with APC and conventional therapies

\begin{tabular}{|c|c|c|c|}
\hline Parameter & $\begin{array}{c}\text { APC plus conventional } \\
\text { therapies }\end{array}$ & Conventional therapies & $P$-value \\
\hline Controlling the rectal bleeding & $41(100 \%)$ & $25(43.1 \%)$ & $<0.004$ \\
\hline Relief of pain around the anus & $34(83.5 \%)$ & $36(62 \%)$ & $<0.36$ \\
\hline
\end{tabular}
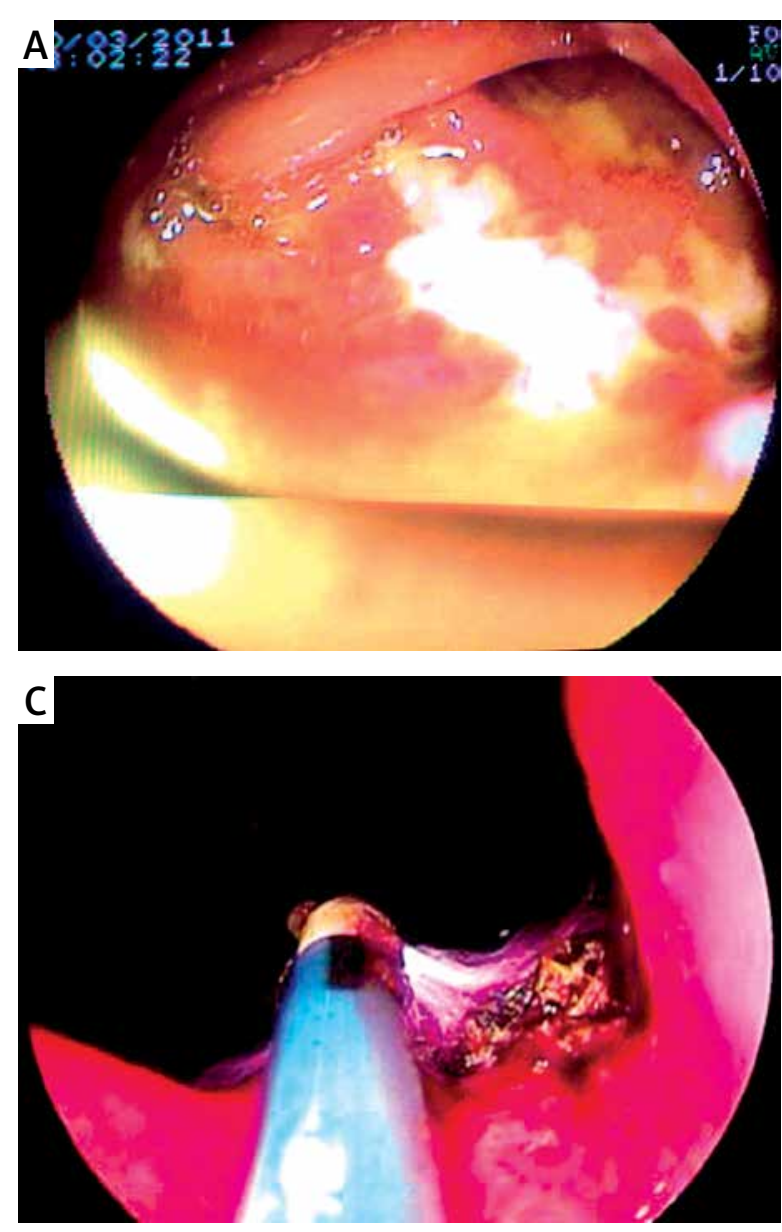

and $70 \%$, respectively. Findings showed that the effectiveness of APC in treating SRUS was higher compared to the conventional therapies $(p<0.001)$. These findings are consistent with those obtained by a study in 2010. They reported the higher effectiveness of APC in treating SRUS, controlling bleeding, and complete ulcer healing compared to conventional therapies $(p<0.036)$ [5]. Our findings are also consistent with the results obtained by Stoppino et al. They used APC to treat SRUS in a 64-year-old female and reported its effectiveness in the treatment of ulcers, reducing bleeding, and pain relief [11]. However, our findings are inconsistent with those reported by Gopal et al. They reported a case in which APC was not effective in treating bleeding polyp-

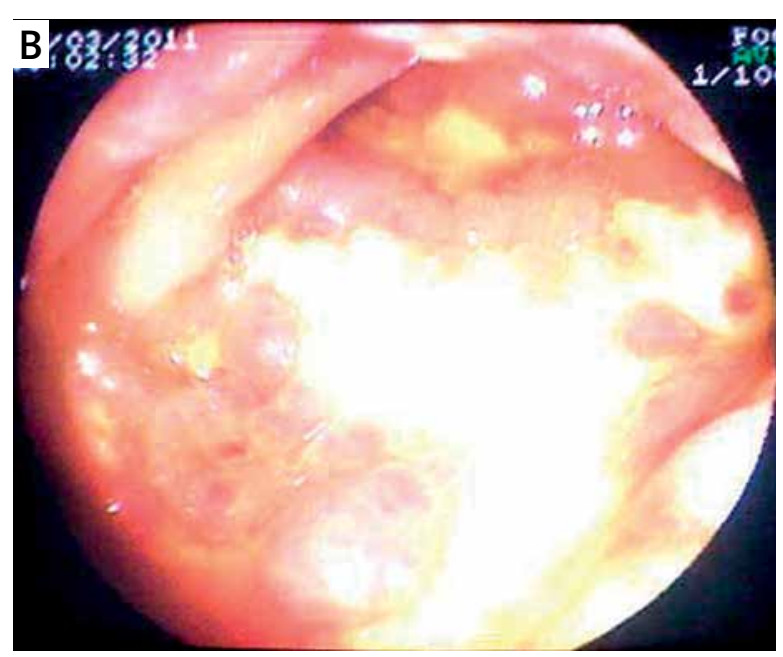

Figure 5. Diagnosed SRUS images: A, B - SRUS, C - APC done for SRUS

oid lesions, and they had to surgically treat the lesion. This increased the uncertainty about the effectiveness of APC in the treatment of bleeding rectal lesions [12]. The results of an experimental study showed that APC may cause injuries in the propria muscularis layer of the experimental animals [13]. In general, few studies reported the superiority of APC plus conventional therapies over conventional therapies alone, which can be due to the low incidence of this disorder in the general population (1 to 5.3 per 100,000 people) [14].

The results showed a significant difference between the two groups in terms of the continuation of healing. The continuation of healing was greater in the APC-treated group compared to those treated with con- 
ventional therapies $(p<0.001)$. This finding is consistent with the results of other studies [5].

The results of this study on better effectiveness of APC compared to conventional therapies in treatment of SRUS showed that APC stops bleeding ( $p<0.023)$. Healing was found to be faster in the group receiving APC plus conventional therapies compared to the group receiving conventional therapies $(p<0.0001)$.

\section{Conclusions}

The present study showed higher effectiveness of APC compared to conventional therapies. Treatment with APC was more effective in healing the rectal ulcers and continuation of healing compared to conventional therapies for SRUS (behavioural therapy, laxatives, and fibre-rich diet). The possibility of healing of bleeding rectal ulcers by treatment with APC was higher than in conventional therapies. Given the low number of studies on the superiority of treatment with APC over conventional therapies, further research in this area is recommended to comment with greater certainty on the effectiveness of APC in all patients with rectal bleeding ulcer.

\section{Conflict of interest}

The authors declare no conflict of interest.

\section{References}

1. Jarrett ME, Emmanuel AV, Vaizey CJ, Kamm MA. Behavioural therapy (biofeedback) for solitary rectal ulcer syndrome improves symptoms and mucosal blood flow. Gut 2004; 53: 368-70.

2. Waniczek D, Rdes J, Rudzki MK, et al. Effective treatment of solitary rectal ulcer syndrome using argon plasma coagulation. Prz Gastroenterol 2014; 9: 249-53.

3. Zhu QC, Shen RR, Qin HL, Wang Y. Solitary rectal ulcer syndrome: clinical features, pathophysiology, diagnosis and treatment strategies. World J Gastroenterol 2014; 20: 738-44.

4. Sharma A, Misra A, Ghoshal UC. Fecal evacuation disorder among patients with solitary rectal ulcer syndrome: a case-control study. J Neurogastroenterol Motil 2014; 20: 531-8.

5. Somani SK, Ghosh A, Avasthi G, et al. Healing of a bleeding solitary rectal ulcer. Gastrointest Endosc 2010; 71: 578-83.

6. Abid S, Khawaja A, Bhimani SA, et al. The clinical, endoscopic and histological spectrum of the solitary rectal ulcer syndrome: a single-center experience of 116 cases. BMC Gastroenterol 2012, 12: 72.

7. Sharara Al, Azar C, Samir S, et al. Solitary rectal ulcer syndrome: endoscopic spectrum and review of the literature. Gastrointest Endosc 2005; 62: 755-62.

8. Dehghani SM, Malekpour A, Haghighat M. Solitary rectal ulcer syndrome in children: a literature review. World I Gastroenterol 2012; 18: 6541-5.
9. Torres C, Khaikin M, Bracho J, et al. Solitary rectal ulcer syndrome: clinical findings, surgical treatment, and outcomes. Int J Colorectal Dis 2007; 22: 1389-93.

10. Zargar SA, Khuroo MS, Mahajan R. Sucralfate retention enemas in solitary rectal ulcer. Dis Colon Rectum 1991; 34: 455-7.

11. Stoppino V, Cuomo R, Tonti P, et al. Argon plasma coagulation of hemorrhagic solitary rectal ulcer syndrome. J Clin Gastroenterol 2003; 37: 392-4.

12. Gopal DV, Young C, Katon RM. Solitary rectal ulcer syndrome presenting with rectal prolapsed, severe mucorrhea and eroded polypoid hyperplasia: case report and review of literature. Can J Gastroenterol 2001; 15: 479-83.

13. Norton ID, Wang L, Levine SA. In vivo characterization of colonic thermal injury caused by argon plasma coagulation. Gastrointest Endosc 2002; 55: 631-6.

14. Haray PN, Morris-Stiff GJ, Foster ME. Solitary ulcer syndrome - an underdiagnosed condition. Int J Colrectal Dis 1997; 12: 213-5.

Received: 27.06 .2015

Accepted: 1.02 .2016 